\title{
Simulation and Improvement of Vehicle Frame Using FEM
}

\author{
Wang Li-rui \\ College of Mechanical and Vehicle Engineering, Hunan \\ University \\ Changsha, China \\ wanglirui91@126.com
}

\author{
Yang Xiao-long \\ College of Mechanical and Vehicle Engineering, Hunan \\ University \\ Changsha, China \\ xyangsuc@163.com
}

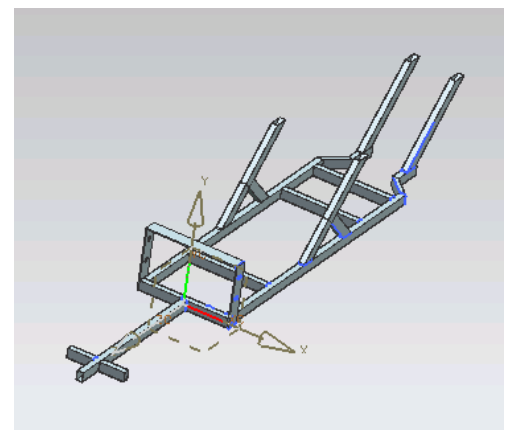

Fig 1 Frame model

In this model, the engine is put in the rear of the frame and the vehicle was driven by the rear wheel (RR). Moreover, to make the frame suitable for the tire with diameter of $305 \mathrm{~mm}$ and make the center of gravity lower, the bearing pedestal was designed in the rear of the frame on the beam of the steering part.

\section{III.Definition and Application of the Load and Boundary Conditions}

The load mainly includes the weights of the engine and the driver, which are $20 \mathrm{~kg}$ and $45 \mathrm{~kg}$, respectively. Acceleration when braking is $2 \mathrm{~m}^{2} / \mathrm{s}$. Acceleration when steering was $6 \mathrm{~m}^{2} / \mathrm{s}$. The safety index was defined as 1.5 according to previous study. The load was applied to the frame where the engine was to be mounted. The sum of the wheel weight and weight of other light components were balanced against the weight to the engine (fig 2).

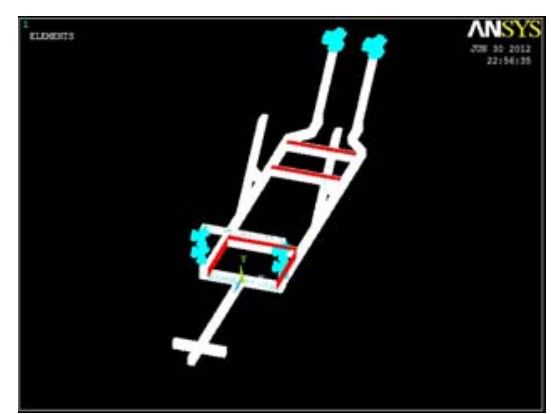

Fig 2 Static load and boundary conditions 
Boundary conditions were restraints on the 4 holes where the front axle and bearing pedestal were to be mounted.

\section{IV.Structural Analysis}

\section{A.Definition of Element type and Material}

Import the model from UG NX6 and use solid 45 element to mesh the whole model. Solid 45 element is designed for 3D solid structure and was defined by 8 nodes. Every node has three degrees of freedom along $\mathrm{x}, \mathrm{y}$ and $\mathrm{z}$ dimensions. This element has the character of plasticity, creepage, expansion, stress strengthening, large deformation and large displacement, and is appropriate for the isotropic materials. For these reasons above, solid 45 element can be used for the analysis of the vehicle frame. 6061 aluminum alloy is an isotropic material. Its Young Modulus is 68.9GPa, Poisson's ratio is 0.33 , yield strength is $55.2 \mathrm{MPa}$ and fatigue strength is $62.1 \mathrm{MPa}$.

\section{B.Meshing of the vehicle frame}

After the element type and material defined, mesh the vehicle frame. Use smart size 5 to mesh the whole vehicle frame and then refine the meshing at key positions. There are 280172 element $s$ meshed in this process.

\section{C.Applying of Load and Solution}

The calculation of the static load is as following procedure: first calculate the space of the truss under the engine which is $15000 \mathrm{~mm}^{2}$, then the pressure on the tress is $13333 \mathrm{~Pa}$, the space of the truss bearing the chair is $20000 \mathrm{~mm}^{2}$, then the pressure on the truss is $25000 \mathrm{~Pa}$. After applying the load(fig 2), solutions were obtained and plotted(fig 3).
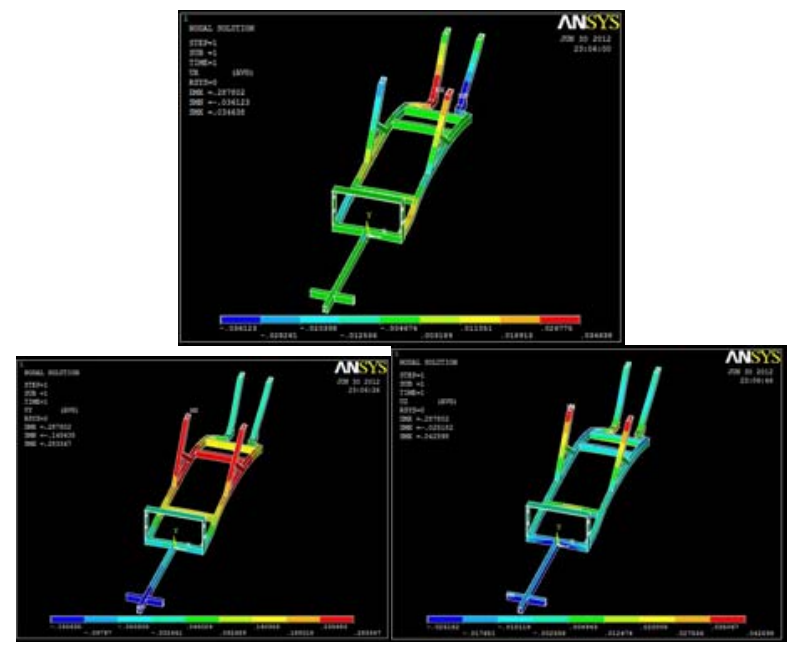

Fig 3 static analysis solutions

As we can tell from the simulation, the displacement from the $\mathrm{X}, \mathrm{Y}$, and $\mathrm{Z}$ dimensions of the frame are $0.03 \mathrm{~mm}, 0.28 \mathrm{~mm}$, $0.04 \mathrm{~mm}$, respectively. The stress of the frame along the $\mathrm{X}, \mathrm{Y}, \mathrm{Z}$ axes are 22.1 MPa, 16.1 MPa and 41.0 MPa, respectively. All of the displacements and stresses are within the limitations ${ }^{[1]}$.

In the dynamic braking simulations, the acceleration was defined as $2 \mathrm{~m}^{2} / \mathrm{s}$. Therefore, the pressure applied on the truss bearing chair and engine are $19200 \mathrm{~Pa}$ and $7111.12 \mathrm{~Pa}$, respectively. After the load was applied, including the weight and acceleration induced pressure on the frame, solutions were obtained and plotted (fig 4).

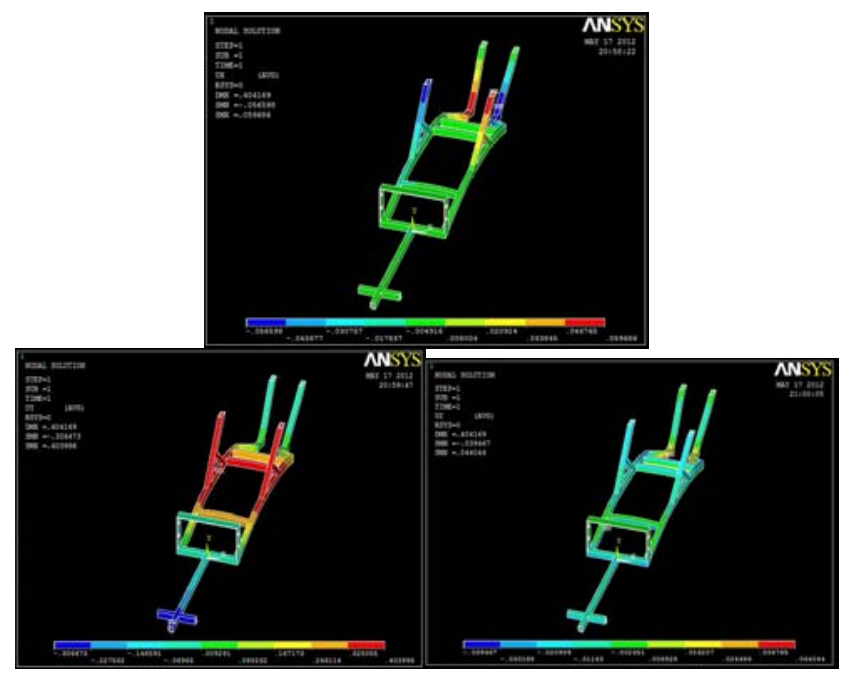

Fig 4 braking condition solutions

As we can tell from the simulation, the displacement from the $\mathrm{X}, \mathrm{Y}$, and $\mathrm{Z}$ dimensions of the frame are $0.059 \mathrm{~mm}, 0.40$ $\mathrm{mm}, 0.044 \mathrm{~mm}$, respectively. The stress of the frame along the $\mathrm{X}, \mathrm{Y}, \mathrm{Z}$ axes are $30.1 \mathrm{MPa}, 22.1 \mathrm{MPa}$ and $59.1 \mathrm{MPa}$, respectively. The maximum stress exceeded the material limitations which means improvements shall be made ${ }^{[2]}$.

In the dynamic steering simulations, the acceleration was calculated as $6 \mathrm{~m}^{2} / \mathrm{s}$. Therefore, the pressure applied on the truss bearing chair and engine are $48000 \mathrm{~Pa}$ and $32000 \mathrm{~Pa}$, respectively. After the load was applied, including the weight and acceleration induced pressure on the frame, solutions were obtained and plotted (fig 5).

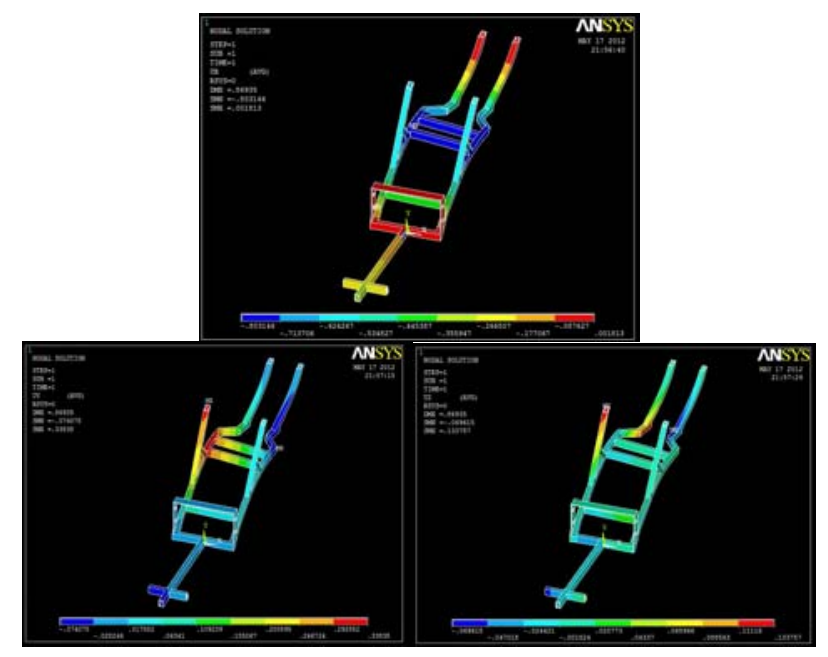

Fig 5 steering condition solutions

As we can tell from the simulation, the displacement from the $\mathrm{X}, \mathrm{Y}$, and $\mathrm{Z}$ dimensions of the frame are $0.803 \mathrm{~mm}, 0.338$ $\mathrm{mm}, 0.133 \mathrm{~mm}$, respectively. The stress of the frame along the $\mathrm{X}, \mathrm{Y}, \mathrm{Z}$ axes are $37.1 \mathrm{MPa}, 36.7 \mathrm{MPa}$ and 60.3MPa, 
respectively. The maximum stress exceeded the material limitations which means improvements shall be made ${ }^{[2]}$.

\section{V.Improvements}

Sine the maximum stress exceeded the material limitations, strengthening components should be designed to strengthen the weak positions(Fig 6). Analysis of it indicates this component will make the maximum displacement and stress within material limitations.

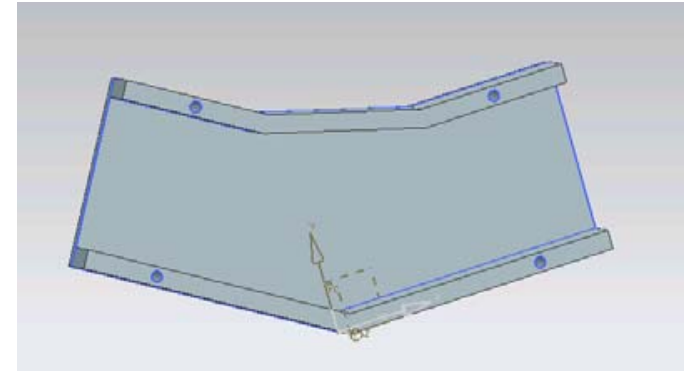

Fig 6 strengthening component

\section{VI.Discussion}

We also used Hyperworks to validate the model using another shell element type algorithm ${ }^{[3]}$. However, the validation shows a slight difference in the displacement in the three dimensions. Although the variation will not influence the structural stability and safety, further validation shall be done to verify the variations.

Moreover, since this vehicle, which is based on the frame, will join the competition to compete for the lowest oil consumption, the frame seems to be a little bit heavy. This means further improvement shall be made if more appropriate materials are available ${ }^{[4][5][6]}$.
The lightweight design and improvement should not be relied only on materials, rather, optimization of components and structure shall be considered when designing ${ }^{[7]}$.

\section{VII.Conclusions}

Simulation using ANSYS 12.0 and Hyperworks has successfully validated the model in static conditions and dynamic conditions with the safety index in mind. Based on the solutions, new component was designed to strengthen the frame. After improvements above, all statistics, including displacement, stress and load distribution are within the material limitations. The model is appropriate for the vehicle to join the competition.

\section{REFERENCES}

[1] Zhou Jianmei, Wang Guijiao, "Lightweight Design of an Energy Conservation Vehicle Frame Based on FEA", J. Tianjin Auto, vol. 9, pp.72 74, 2008

[2] Wang Guijiao, Zhou Jianmei, “The Type Selecting and Lightweight Designing of Energy-saving Vehicles’ Frame”, J. Auto Technologies, vol. 9, pp. 41 44, 2008

[3] Dong Xueqin, Xin Yong, Yang Fan, "On Finite Element Modeling of a Vehicle’s Frame Using Hyperworks”, J. Mechanical Science and Technology for Aerospace Engineering, vol. 7, pp.906 908, 2008

[4] Gao Yunkai, “ Analysis of a Vehicle Body”, Beijing Institute of Technology Press, 2006

[5] Xia Xiaokun, Yan Fuwu, Du Changqing, “A Study on Reducing Oil Consumption of Energy-efficient Motorcycle”, J. Motorcycle Technologies, vol. 10, pp.70 72, 2008

[6] Yin Huijun, Wei Zhilin, Shen Guanglie, "Finite Element Analysis on Frame of Trucks”, J. Journal of Machine Design, vol. 11, pp.27 28, 2005

[7] Li Xiya, Li Chenggang, Hu Yujin, "General Conditions of the Finite Element Analysis Technic”, J. Special Purpose Vehicle, vol. 1, pp.11 15, 2001 